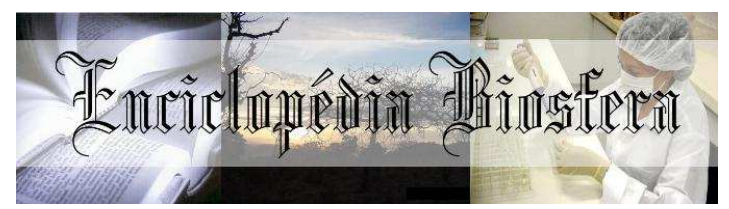

\title{
SEGMENTO CAUDAL DA ARTÉRIA AORTA ABDOMINAL E SUAS RAMIFICAÇÕES EM QUATI (Nasua nasua) Linnaeus, 1766
}

Rodrigo Lopes de Felipe ${ }^{1}$, Duvaldo Eurides ${ }^{2}$, Daniela Cristina de Oliveira Silva ${ }^{3}$, Zenon Silva ${ }^{4}$, Eduardo Paul Chacur ${ }^{4}$, Roseâmely Angélica de Carvalho Barros ${ }^{4}$ Mônica Duarte da Silva ${ }^{5}$,Dara Rúbia Souza Silva ${ }^{5}$.

'Doutorando da Universidade Federal de Uberlândia. Campus Umuarama. 38400-902, Uberlândia, MG, Brasil, rlfarmaceutico@bol.com.br.

${ }^{2}$ Docente da Pós Graduação em Medicina Veterinária da Universidade Federal de Uberlândia. Campus Umuarama.

${ }^{3}$ Docente do Instituto de Ciências Biomédicas (Disciplina de Anatomia Humana) da Universidade Federal de Uberlândia.

${ }^{4}$ Docente do Instituto de Biotecnologia Núcleo de Ciências Biológicas, Universidade Federal de Goiás - Regional Catalão, Catalão - GO.

${ }^{5}$ Graduando do Curso de Ciências Biológicas, Universidade Federal de Goiás - Regional Catalão, Catalão - GO.

Recebido em: 02/10/2017 - Aprovado em: 21/11/2017 - Publicado em: 05/12/2017 DOI: 10.18677/EnciBio_2017B90

\begin{abstract}
RESUMO
O quati é um mamífero da ordem carnívora, família procionídae e gênero Nasua sendo considerado um animal fora de riscos de extinção, entretanto, o controle da população deve ser monitorado assim como somar estudos sobre a biologia da espécie. O sistema arterial é importante para a manutenção da vida, e conhecê-lo bem, assim como identificar as possíveis diferenças anatomicas é relevante para as práticas anatômofisiológicas e clínicas. Objetivou-se realizar um estudo sobre o seguimento caudal da artéria aorta abdominal e suas ramificações em quati. Foram dissecados três espécimes, após a injeção de látex Arte Cola corado e fixação com solução de formol a 10\%. O seguimento caudal da artéria aorta abdominal de quati emite ramos colaterais e ramos terminais. Os ramos colaterais incluem um par de artérias lombares, uma artéria mesentérica caudal, um par de artérias circunflexas ilíacas profundas e um par de artérias ilíacas externas. Os ramos terminais compreendem um par de artérias ilíacas internas e uma artéria sacral mediana. 0 padrão de ramificação, e distribuição guarda similitude com as descrições literárias em cão doméstico.
\end{abstract}

PALAVRAS-CHAVE: anatomia veterinária, procionídeos, vasos sanguíneos.

\section{THE CAUDAL SEGMENT OF THE ABDOMINAL AORTA ARTERY AND ITS RAMIFICATIONS IN COATI (Nasua nasua) Linnaeus, 1766}

\footnotetext{
ABSTRACT

The quati is a mammal of the carnivorous order, family of the procionids and genus Nasua being considered an animal without risks of extinction, however, the control of the population must be monitored as well as to differsify studies on the species. The arterial system of animals is important for maintaining its vitality, and knowing it as
} 
well as its possible differences is relevant to anatomical and clinical practices. The objective of this study was to study the caudal follow-up of the abdominal aorta and its ramifications in the coati. Three specimens were dissected after dye injection and fixation with formaldehyde. The caudal follow-up of the abdominal aorta of the coati, emits collateral branches and terminal branches. The collateral branches include a pair of lumbar arteries, a caudal mesenteric artery, a pair of deep iliac circumflex arteries, and a pair of external iliac arteries. The terminal branches comprise a pair of internal iliac arteries and a median sacral artery. The pattern of branching, and distribution was similar to that described in the dog.

KEYWORDS: procionids, blood vessels, veterinary anatomy.

\section{INTRODUÇÃO}

A artéria aorta foi descrita por Getty (2008), como a principal via de fornecimento de sangue arterial para o corpo de carnívoros, sendo que após cruzar o hiato aórtico é denominada artéria aorta abdominal. Em seu seguimento caudal emite ramos tanto colaterais quanto terminais (DYCE et al., 2004). Disfunções em suas ramificações podem significar várias condições clínicas (CARVALHO et al., 2008) e conhecer as variações do padrão de vascularização visceral é relevante para as práticas radiográficas e cirúrgicas (ESTRUC et al., 2012).

O estudo da morfologia de animais silvestres pode contribuir para elucidação de dúvidas e questionamentos sobre os seus sistemas orgânicos. Um destes animais distribuído amplamente no Brasil, componente de vários biomas é o quati (Nasua nasua), que pertence a ordem carnívora e família procionidae (BEISIEGEL, CAMPOS, 2013). Apesar de ser considerado uma espécie não ameaçada de extinção (BEISIEGEL; CAMPOS, 2013), em áreas de caça, o número de especimes tende a diminuir sendo ainda descrito como componente da fauna atropelada em rodovias (GOMES et al., 2013). Apesar de ser uma espécie comum, a biologia do quati carece de estudos mais detalhados (BEISIEGEL; CAMPOS, 2013). Objetivouse descrever o seguimento caudal da artéria aorta abdominal e suas ramificações, no quati (Nasua nasua) Linnaeus, 1766.

\section{MATERIAL E MÉTODOS}

Foram utilizadas três carcaças de quati adultos, porém, de idade desconhecida, e peso indefinido, sendo dois machos e uma fêmea, dois espécimes foram recolhidos mortos às margens de rodovias que cruzam o sudeste goiano, e um espécime foi doado pelo Instituto Brasileiro do Meio Ambiente e dos Recursos Naturais Renováveis (IBAMA).

Os espécimes foram congelados para conservação e posteriormente descongelados em água corrente. A artéria carótida comum esquerda foi canulada com uma sonda uretral número oito, para injeção de látex Arte Cola corado com pigmento vermelho. Em seguida foram perfundidos no sistema venoso com solução de formol a $10 \%$, para fixação e conservados em submersos em igual solução, até os procedimentos de dissecação. A técnica utilizada foi a proposta por Walker, (1980). Esta pesquisa conta com a autorização para atividades de pesquisa (SISBIO - 37072-2), e aprovação do comitê de ética em pesquisa com animais (CEUA UFU, 067/12). Os achados foram denominados em analogia com estruturas descritas em carnívoros domésticos, em conformidade com a Nomina Anatômica Veterinária (I.C.V.G.A.N 2012). 


\section{RESULTADOS E DISCUSSÃO}

Neste artigo, considera-se como seguimento caudal da artéria aorta abdominal a porção correspondente ao seu terço caudal. O segmento caudal da artéria aorta abdominal deriva ramos colaterais e terminais. Os colaterais destinamse à parede abdominal e estruturas anatômicas circunvizinhas, sendo um par de artérias lombares, uma artéria mesentérica caudal, um par de artérias circunflexas ilíacas profundas, um par de artérias ilíacas externas, e os ramos terminais, um par de artérias ilíacas internas e uma artéria sacral mediana (Figura 1).

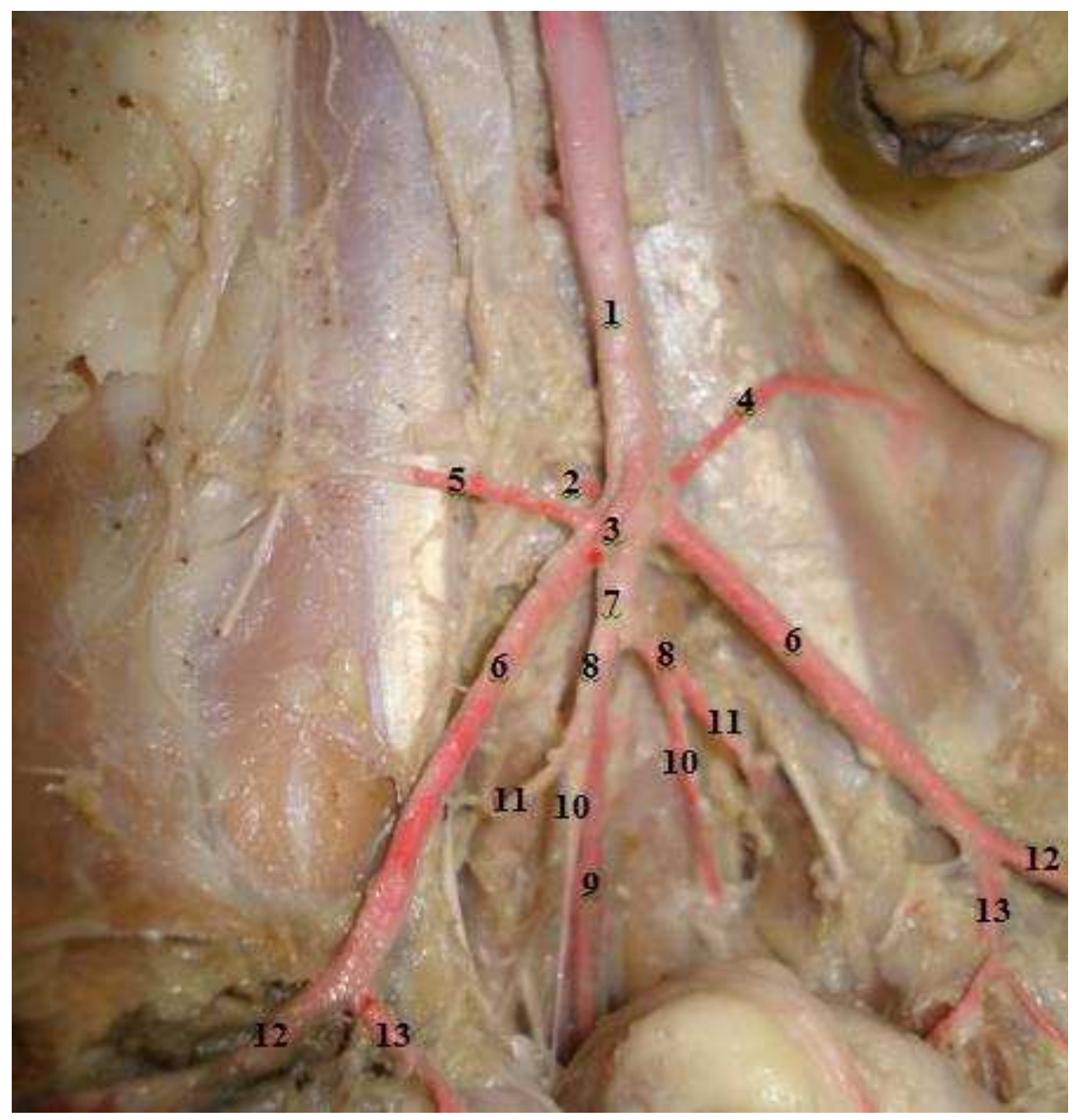

Seguimento caudal da artéria aorta (1), artéria lombar direita (2), artéria mesentérica caudal (3), artéria circunflexa ilíaca profunda esquerda (4), artéria circunflexa ilíaca profunda direita (5), artérias ilíacas externas, direita e esquerda (6), porção terminal do seguimento caudal da artéria aorta abdominal (7), artérias ilíacas internas direita e esquerda (8), artéria sacral mediana (9), ramos viscerais (10), ramos obturatórios (11), artérias femorais direita e esquerda (12), artérias femorais profundas direita e esquerda (13). Fonte: os autores.

FIGURA 1. Padrão de ramificação da parte caudal da artéria aorta abdominal do quati.

A descrição do seguimento caudal da artéria aorta abdominal difere, significativamente das citações, em cães, por Dyce et al. (2004), Getty, (2008) e Ghoshal, (2008), quando afirmaram que a porção caudal emite as artérias ilíacas 
externas e internas, além da sacral mediana, uma vez que, em quati, nascem as artérias ilíacas externas, e segue um tronco comum para as ilíacas internas e sacral mediana. Além disso, outros ramos colaterais estão presentes, tais como artérias lombares, circunflexa ilíaca profunda esquerda e mesentérica caudal.

Artérias lombares são os primeiros ramos oriundos da parte terminal da aorta abdominal. Eles nascem pela face dorsal, ou dorsolateral da aorta, próximo à origem da artéria circunflexa ilíaca profunda esquerda e logo após penetraram nos músculos relacionados à estabilização da coluna vertebral (região lombar).

Em quati, assim como em cão, conforme referido por Dyce et al. (2004), as artérias lombares surgem como ramos colaterais da aorta e irrigam estruturas parietais no dorso. A descrição da origem na face aórtica dorsal, da artéria lombar esquerda não foi encontrada na literatura consultada. Ghoshal (2008), descreveu, em cão, sete pares de artérias lombares derivadas ao longo de toda a artéria aorta. Nesta pesquisa, é descrito apenas o último par de artérias lombares, cuja origem ocorre no segmento caudal. Por outro lado, Dyce, (2004), Getty, (2008) não citaram quantos pares de artérias lombares se originam na porção caudal da aorta.

Em quati, a artéria mesentérica caudal que se origina na superfície ventral do segmento caudal da aorta, cranialmente à origem das artérias ilíacas externas é ímpar, e destina-se à parede do colo descendente, suprindo o segmento caudal do intestino grosso. Em cão e no gato Silva et al. (2007), Getty (2008), e Ghoshal, (2008), apresentaram a mesma característica de origem e distribuição da artéria mesentérica caudal observada, nesta pesquisa, em quati, qual seja, origem a partir da superfície ventral da aorta e distribuição na superfície do cólon descendente. Schaller, (1996) relatou que, em pequenos animais a referida artéria é estritamente visceral, irrigando a parte caudal do intestino grosso, fato também observado em quati. Em jaguatirica, segundo Pinheiro et al. (2014), ocorre o mesmo padrão de origem e distribuição observado em quati. Várias patologias podem afetar o intestino, como torções, intuscepção, tumores e isquemia, daí a importância de se conhecer o padrão de vascularização intestinal, visando um atendimento clínico de qualidade (RAHAL et al., 2000; ZERBIB et al., 2008; URBIZTONDO et al., 2010).

Em quati, a artéria circunflexa ilíaca profunda esquerda apresenta duas modalidades diferentes de origem. Na primeira, que ocorrem em dois, exemplares, ocorre na superfície dorsolateral da artéria aorta, cranial à origem da artéria mesentérica caudal, corre transversalmente para o flanco esquerdo, cruzando o músculo psoas maior até alcançar a superfície profunda da parede dorsolateral do abdome. Na segunda modalidade ocorrente em um exemplar, sua emergência ocorre caudalmente à origem da artéria mesentérica caudal. $O$ primeiro padrão de origem da artéria circunflexa ilíaca profunda esquerda em quati é semelhante ao descrito, em cão por Dyce et al. (2004), Ghoshal, (2008) e Getty, (2008). O segundo padrão não foi descrito na literatura de animais domésticos.

Em relação ao trajeto e distribuição, ambos os padrões encontrados em quati são semelhantes às descrições, em cão (DYCE et al., 2004). Artéria circunflexa ilíaca profunda direita, em quati, origina da superfície dorsolateral da artéria ilíaca externa, e corre crânio-lateralmente, cruzando a superfície ventral do músculo psoas maior na direção da parede dorsolateral do abdome, na qual se ramifica. Esta origem, apesar de não ser o padrão normalmente encontrado em cão, foi referido por Ghoshal, (2008). Alguns casos, tanto a circunflexa esquerda quanto a direita podem se originar da artéria ilíaca externa, mas, neste trabalho, foi observada apenas no antímero direito, porém, em todos os espécimes dissecados. 
O local de origem das artérias circunflexas ilíacas profundas, em cão é descrito, a partir do mesmo nível de origem da artéria mesentérica caudal (GOSHAL, 2008), condição não observada neste trabalho. A circunflexa esquerda origina cranial acima da artéria mesentérica caudal, em dois espécimes e abaixo dela, em um espécime. Por sua vez a direita nasce da artéria ilíaca externa nos três espécimes, sem relação direta com a mesentérica caudal.

Em quati, as artérias llíacas Externas, direita e esquerda surgem como principais ramos da parte caudal da artéria aorta abdominal, pela face lateral da mesma. Estas artérias correspondem a dois grandes ramos arteriais que podem nascer dicotomicamente na mesma altura, o que ocorreu em dois espécimes ou a esquerda apresentar uma origem mais cranial em relação à direita, o que ocorreu em um espécime.

As artérias correm caudolateralmente sobre a borda da pelve menor, posicionando-se obliquamente ao vaso principal, seguindo na direção do anel inguinal. Ao atravessar o canal inguinal, e deixando a cavidade abdominal passando a denominarem-se artérias femurais. A artéria ilíaca externa esquerda não emite nenhum ramo colateral, porém a ilíaca externa direita emite craniolateralmente a artéria circunflexa ilíaca profunda direita.

Assim como verificado em quati, em cão Dyce et al. (2004) e Ghoshal (2008), descreveram um par de artérias ilíacas externas como derivadas do segmento caudal da aorta. As artérias femurais e femurais profundas que irrigam o membro pélvico em quati, são ramificações terminais das ilíacas externas, condição igualmente descrita, em cão, por Dyce et al. (2004) e Ghoshal, (2008).

Segundo Pinheiro et al. (2014), em jaguatirica as artérias ilíacas externas surgem da face lateral da aorta abdominal, semelhante condição é observada em quati. Foi observado também que em quati, a artéria ilíaca externa, ainda na cavidade abdominal, emite a artéria femoral, como relatado por Dyce et al. (2004) e Ghoshal, (2008) em cão.

Verifica-se que, em quati, o segmento caudal da artéria aorta abdominal, após a origem das artérias ilíacas externas, sofre uma redução de calibre, e uma torção dorsolateral direita, seguindo distalmente por curta distância levemente deslocada à direita da linha sacral mediana e justaposta à superfície ventral do sacro, se comportando como um tronco arterial antes de emitir os três ramos terminais: as artérias ilíacas internas direita e esquerda e artéria sacral mediana, condição não descrita na literatura pertinente. Por outro lado, em jaguatirica, Pinheiro et al. (2014) citaram que artéria aorta abdominal termina formando as artérias ilíacas internas e externas além da sacral mediana.

As observações, em quati, mostraram que as artérias ilíacas internas, direitas e esquerdas surgem da porção terminal da aorta abdominal e correm caudolateralmente sobre a borda da pelve menor, e em seu trajeto emitem pequenos ramos viscerais para estruturas intrapélvicas e parede pélvica, além de um ramo obturatório. Essa condição está de acordo com as descrições de Dyce et al. (2004) e Ghoshal, (2008) em cão e por Carvalho et al. (2008), em pequenos animais.

Ainda, em quati, artéria sacral mediana, ultimo ramo da artéria aorta abdominal, representa a continuação caudal desta, que após a sua origem corre caudalmente sobre a superfície ventral do sacro, cóxi e depois da cauda, suprindo os músculos e outras estruturas da cauda, através de ramos emitidos dorsalmente. Dyce et al. (2004) e Ghoshal, (2008) referiram que em cão a sacral mediana é uma 
continuação da artéria aorta abdominal, porém de calibre menor que irriga estruturas caudais.

\section{CONCLUSÃO}

O padrão básico de ramificação da parte terminal da aorta em quati e em cão mostra similitudes, porém, pequenas diferenças inerentes à espécie podem ser observadas, quanto à origem das artérias colaterais assim como ramos terminais.

\section{REFERÊNCIAS}

BEISIEGEL, B. M; CAMPOS, C. B. Avaliação de risco de extinção do Quati Nasua nasua (Linnaeus, 1766), no Brasil. Biodiversidade Brasileira, v.3 n.1, p.169-276, 2013.

http://www.icmbio.gov.br/revistaeletronica/index.php/BioBR/article/view/396/337> Acesso em: 16 de Out. 2016.

CARVALHO, C.F; CHAMMAS, M.C; CERRI, G.G. Morfologia duplex Doppler dos principais vasos sanguíneos abdominais em pequenos animais. Ciência Rural, v.38, n.3, p.880-888, 2008. Disponível em:< http://www.scielo.br/pdf/cr/v38n3/a48v38n3.pdf> Acesso em: 16 de Out. 2016.

DYCE, K.M.; SACK, W.O.; WENSING, C.J.G. Tratado de Anatomia Veterinária. 3.ed. Rio de Janeiro: Elsevier, 2004. 813p.

ESTRUC, T.M; NASCIMENTO, R.M; GOMES, M.S; MENCALHA, R; ABIDUFIGUEIREDO, M. Relações anatômicas entre a origem e distribuição das artérias mesentéricas cranial e caudal no gato doméstico. Revista Brasileira de Medicina Veterinária, v.34, n.4, p.295-302, 2012. Disponível em: <http://www.rbmv.com.br/pdf_artigos/21-12-2012_10-06RBMV\%20008.pdf> Acesso em 17 de Out. 2016.

GETTY, R. Generalidades sobre o coração e os vasos sanguíneos. In: SISSON \& GROSSMAN. Anatomia dos animais domésticos. Vol. 1, 5.ed. Rio de Janeiro: Guanabara Koogan, 2008. 2000p. Cap. 11.

GHOSHAL, N. G. Coração e artérias do carnívoro. In: SISSON \& GROSSMAN Anatomia dos animais domésticos. Vol. 2, 5.ed. Rio de Janeiro: Guanabara Koogan, 2008. 2000p. Cap. 55.

GOMES, D.C; SILVA, C.V; FARIA, A.A; MORAIS, M.A.V; SANT'ANA, C.E.R; MENDONÇA, L.G.A. Registro de atropelamento de animais silvestres entre as cidades de Palmeiras de Goiás e Edealina, GO. Revista Eletrônica Interdisciplinar, v.10, n.1, p.19-34, 2013. Disponível em:< http://revista.univar.edu.br/index.php/interdisciplinar/article/view/20> Acesso em: 16 de Out. 2016.

I.C.V.G.A.N. (International Committee On Veterinary Gross Anatomical Nomeclature). Nômina Anatômica Veterinária. 15.ed. Columbia: Committee Hannover, 2012. 166p. 
PINHEIRO, L.L; ARAÚJO, E.B; LIMA, A.R; MARTINS, D.M; MELUL, R; SOUZA, A.C.B; PEREIRA, L.C; BRANCO, E. Os ramos colaterais da aorta abdominal em jaguatirica (Leopardus pardalis). Pesquisa Veterinária Brasileira, v.34, n.5, p.491495, 2014. Disponível em: < http://www.scielo.br/pdf/pvb/v34n5/v34n5a18.pdf> Acesso em: 15 de Out. 2016.

RAHAL, S.C; GARIB, M.I; MAMPRIM, M.J; TEIXEIRA, C.R. Mesenteric torsion in a dog. Canadian Veterinary Journal. v.41, p.710-711, 2000. Disponível em: $<$ https://www.ncbi.nlm.nih.gov/pmc/articles/PMC1476402/pdf/canvetj000210056.pdf> Acesso em: 17 de Out. 2016.

SCHALLER, O. Nomenclatura anatômica veterinária ilustrada. Zaragoza: Acríbia, 1996. $614 \mathrm{p}$.

SILVA, R.M; WANDERLEY, L.S; MOTTA, A.L.A. Origem e distribuição da artéria mesentérica caudal em gatos sem raça definida. Arquivos de Ciências Veterinárias e Zoologia da Unipar, v.10, n.2, p.121-124, 2007. Disponível em: <http://189.126.110.61/acvzunipar/article/view/13580/14450> Acesso em 16 de Out. 2016.

URBIZTONDO, R; CHAPMAN, S; BENJAMINO, K. Primary mesenteric root osteosarcoma in a dog. Veterinary Clinical Pathology, v.39, n.3, p.377-389, 2010. Disponível em: <https://www.ncbi.nlm.nih.gov/pubmed/20646259> doi: 10.1111/j.1939-165X.2010.00239.x Acesso em: 16 de Out. 2016.

WALKER, W. F. Vertebrate dissection. 6.ed. Philadelphia: W.B Saunders Company, 1980. 425p.

ZERBIB, P; LEBUFFE, G; SERGENT-BAUDSON, G; CHAMATAN, A; MASSOUILLE, D; LIONS, C; CHAMBON, J.P. Endovascular versus open revascularization for chronic mesenteric ischemia: a comparative study. Langenbeck's Archives of Surgery, n.393, p.865-870, 2008. Disponível em:< http://link.springer.com/article/10.1007/s00423-008-0355-x> Acesso em: 15 de Out. 2016. 